\title{
A HYBRID DRIFT DIFFUSION MODEL: DERIVATION, WEAK STEADY STATE SOLUTIONS AND SIMULATIONS
}

\author{
SIMONE CHIARELLI, FEDERICA DI MICHELE AND BRUNO RUBINO
}

\begin{abstract}
In this paper we derive a new hybrid model for drift diffusion equations. This model provides a description of the quantum phenomena in the parts of the device where they are relevant, and degenerates to a semiclassical model where quantum effects are negligible, so that the system can be considered classically. The study of quantum correction to the equation of state of an electron gas in a semiconductor with the assumption of localized quantum effects leads to a further condition on the classical-quantum interface. Moreover, we prove the existence of weak solutions for our hybrid model. Finally, we present numerical results for different devices, by means of Colsys software.
\end{abstract}

\section{INTRODUCTION}

Over the last years the constant progress of the computer industry has led to the production of electronic devices of nanometer-sized. Reaching the decanano length scale, quantum effects cannot be negligible, thus the widely used classical semiconductor models become inapplicable. To overcome this problem, much effort has been spent to incorporate the relevant quantum mechanical phenomena into the well understood classical model. Recently, the quantum hydrodynamic equations (QHD) and quantum drift diffusion equations (QDD) have been used in order to describe the modern quantum device. In one space dimension the (scaled) QHD and QDD equations for the charge density $n$ and for the current density $J$ are

$$
\left\{\begin{array}{l}
n_{t}+J_{x}=0 \\
J_{t}+\left(\frac{J^{2}}{n}+n T\right)_{x}-n V_{x}-\delta^{2}\left(n(\ln n)_{x x}\right)_{x}=-\frac{J}{\tau}
\end{array}\right.
$$

and

$$
\left\{\begin{array}{l}
n_{t}+J_{x}=0, \\
J=n F_{x}, \\
-\delta^{2} \frac{\sqrt{n}_{x x}}{\sqrt{n}}+T \ln x+V=F .
\end{array}\right.
$$

$M S C$ (2010): primary 34K06, 34K25; secondary 39.

Keywords: drift diffusion, quantum drift diffusion, hybrid model, transmission conditions.

This research was supported by the Research Project MIUR-PRIN 2009 Systems of Conservation Laws and Fluid Dynamics: Methods and Applications. 
Both of them are usually coupled with Poisson equation:

$$
-\lambda^{2} V_{x x}=n-C(x) .
$$

Here $V, T, \tau, \lambda$ are the electrical potential, scaled electron temperature, scaled relaxation time and scaled Debye length, respectively. Moreover, $C(x) \in L^{2}(\Omega)$ is the doping profile describing the fixed charge background ions in a semiconductor crystal. These equations have been extensively studied in the last fourteen years. These models can be derived from a moment expansion of the Wigner-BoltzmannPoisson system [9]. Depending on the number of moments considered, there exists a whole hierarchy of macroscopic quantum models leading from the quantum hydrodynamic model over quantum energy transport to the quantum drift diffusion model [14, 13]. See also [10] and [12] for a complete review of mathematical models for semiconductor. The main difficulty that one has, when using quantum equations, is that they are computationally expensive. But a in very large number of applications, the quantum effects are important in a limited region, e.g. around the double barrier in resonant tunneling diodes, whereas the rest of the device is well described by classical models. Therefore, we develop a hybrid model that provides a complete quantum description wherever necessary but behaves in classical way where the quantum effects are negligible.

In this context the word hybrid indicates a strategy for which we describe a small part of the device by using the quantum models (e.g. Schrödinger equation, QHD or HDD), and the rest using classical models, for example hydrodynamical (HD) or drift diffusion (DD). The problem of the transmission conditions at the interface, between classical and quantum system, is still an open problem. In [4] the authors consider a one-dimensional coupled stationary Schrödinger drift diffusion without collision, in order to link quantum zone and classical zone supposing that the current density is continuous. In [5] the author discusses the transmission conditions between a classical transport model described by the Boltzmann equation and a quantum model described by a set of Schrödinger equations. It is known that, from the numerical point of view, the Schrödinger equation is very difficult to treat and the drift diffusion equations do not provide good results for modeling modern semiconductor devices.

In this paper we study a hybrid model for quantum and classical drift diffusion, both from the numerical and the theoretical point of view. In particular, in Section 2 we derive a hybrid model heuristically, that we shorten H-QDD-DD. In Section 3 we prove the existence of weak solutions, while Section 4 is devoted to numerical tests on some simple devices.

We consider the stationary problem for unipolar device

$$
\left\{\begin{array}{l}
-\delta^{2} \frac{\Delta \sqrt{n}}{\sqrt{n}}+h(n)+V=F \\
\nabla \cdot(n \nabla F)=0
\end{array}\right.
$$

coupled with Poisson equation

$$
-\lambda^{2} \Delta V=n-C,
$$

where $h(n)$ is the enthalpy function, and, in particular, $h(n)=T \ln n$ if the system is in isothermal conditions. 
Here (1.1)-(1.2) are already scaled and the dimensionless physical parameters are the Planck constant $\delta$ and the Debye length $\lambda$. The generalized chemical potential $F$ is linked to current density by $J=n \nabla F$. We note that for $\delta=0$ we obtain the classical drift diffusion, while for $\delta>0$ the quantum correction term

$$
\delta^{2} \frac{\Delta \sqrt{n}}{\sqrt{n}},
$$

this is the so-called Bohm potential.

Basically, our idea is to introduce a smooth function $G_{\alpha}(x)$ that multiplies the quantum correction, which is zero where the quantum effect can be neglected and is otherwise equal to one. We assume $\Omega \subset \mathbb{R}^{N}, N=1,2,3$, is the bounded device domain, and suppose the boundary $\partial \Omega=\Gamma_{D}+\Gamma_{N}$, where $\Gamma_{D} \cap \Gamma_{N}=\emptyset$. In particular, $\Gamma_{D}$ models the Ohmic contacts and $\Gamma_{N}$ represents the insulating parts of the device. Their structure depends on the design of the particular device we are studying. Moreover, we assume $\partial \Omega \in C^{0,1}$, and that $\Gamma_{D}$ has non vanishing $(N-1)$-dimensional Lebesgue measure. We divide the domain $\Omega$ into two disjoint subsets: a classical part $\Omega_{c}$ and a quantum part $\Omega_{q}$, and we set

$$
H(x)= \begin{cases}1 & x \in \Omega_{q}, \\ 0 & x \in \Omega_{c}\end{cases}
$$

and $G_{\alpha}(x)$ its regularization built as follows:

$$
G_{\alpha}(x)=\left(H * \eta_{\alpha}\right)(x):=\int_{\Omega} H(x-y) \eta_{\alpha}(y) \mathrm{d} y,
$$

where $\eta_{\alpha}$ is a smoothing kernel (mollifier) and $\alpha \in(0,1]$ is the regularization parameter. If $\alpha \rightarrow 0+$, then $G_{\alpha}(x) \rightarrow H(x)$.

Except Subsection 3.3, we work with a given $\alpha$ : for sake of simplicity, we take $\alpha=1$ and, to simplify the notation, we set $G_{\alpha=1}(x)=G(x)$.

\section{Quantum correction to the equation of state OF AN ELECTRON GAS IN A SEMICONDUCTOR}

In [2] a macroscopic description of conduction electrons in a semiconductor was presented and the state equation for the electron gas was generalized to include a dependence on the gradient of the density. This generalization leads to a new transport equation, which can be expressed as a generalized drift diffusion current equation that has been shown to describe some important quantum mechanical effects occurring in semiconductor structures.

On a macroscopic scale, the lowest-order effect of quantum mechanics on an electron gas is to make its equation of state density-gradient dependent (see [1]). Basically, we suppose that the energy of the electrons depends on $\nabla n$ in a given part of our device, in which the quantum effects are important, whereas the remaining part can be treated classically. The equation of linear momentum balance in the electron gas under the assumption of negligible inertia may be written as

$$
-\nabla(V+F)+E^{e}=0,
$$


where $V$ is the electric potential, $E^{e}$ is the drag force, $F$ is a generalized chemical potential. For $E^{e}$ the lowest order form is

$$
E^{e}=-\frac{v}{\mu},
$$

which represents an assumption of linear drag where $\mu$ is the usual electron mobility, and $v$ the velocity of the gas. To include lowest-order quantum effects, the equation of state must depend not only on the gas density but also on the density gradient. Thus we can write the internal energy density of the electron gas as:

$$
\xi=\xi(n, \nabla n) .
$$

The expression for the generalized chemical potential $F$ in terms of $\xi$ is:

$$
F=\frac{\partial(n \xi)}{\partial n}-\nabla\left[n \frac{\partial \xi}{\partial \nabla n}\right]
$$

We assume that $\xi$ depends on $n$ and on $G(x) \nabla n$ where $G(x)$ describes how the internal energy depends on gradient of the charge density:

$$
\xi(n, \nabla n)=\ln n+G(x) \frac{\varepsilon^{2}}{2} \frac{(\nabla n)^{2}}{n^{2}} .
$$

Now we put (2.2) into (2.1). The first term on the right side of (2.1) is given in [1], and it reads

$$
\frac{\partial(n \xi)}{\partial n}=(\ln n+1)+\frac{\varepsilon^{2}}{2} G(x) \frac{\nabla n^{2}}{n^{2}} .
$$

The second one, which includes the quantum effects, is

$$
\nabla\left[n \frac{\partial \xi}{\partial \nabla n}\right]=\nabla\left[n \frac{\partial}{\partial \nabla n}\left(\ln n+\frac{\varepsilon^{2}}{2} G(x) \frac{(\nabla n)^{2}}{n^{2}}\right)\right],
$$

where

$$
\begin{aligned}
& \nabla\left[n \frac{\partial}{\partial \nabla n}(\ln n)\right]=1, \\
& \nabla\left[n \frac{\partial}{\partial \nabla n}\left(+\frac{\varepsilon^{2}}{2} G(x) \frac{(\nabla n)^{2}}{n^{2}}\right)\right]=+\varepsilon^{2} G(x)\left(\frac{\Delta n}{n}-\frac{(\nabla n)^{2}}{n^{2}}\right)+\varepsilon^{2} G^{\prime}(x) \frac{\nabla n}{n} .
\end{aligned}
$$

Then we have

$$
\nabla\left[n \frac{\partial \xi}{\partial \nabla n}\right]=1+\varepsilon^{2} G(x)\left(\frac{\Delta n}{n}+\frac{(\nabla n)^{2}}{n^{2}}\right)-G^{\prime}(x) \varepsilon^{2} \frac{\nabla n}{n},
$$

so if we sum (2.3) and (2.4), we find the potential

$$
F=\ln n-\varepsilon^{2} G(x)\left(\frac{\nabla n}{n}-\frac{1}{2} \frac{(\nabla n)^{2}}{n^{2}}\right)-\varepsilon^{2} G^{\prime}(x) \cdot \frac{\nabla n}{n}
$$

and using $\rho=\sqrt{n}$ we get

$$
F=\ln n-2 \varepsilon^{2} G(x)\left(\frac{\Delta \rho}{\rho}\right)-G^{\prime}(x) \varepsilon^{2} \frac{\nabla \rho}{\rho} .
$$




\section{Weak solution to the Hybrid Drift Diffusion model}

We are trying to build a Hybrid Drift Diffusion (HDD) model consisting in a model that includes a part with classical behavior and a part with quantum behavior. For this propose we include a space dependence function $G(x)$ in the quantum correction term. We use the techniques proposed in [7], where the authors prove the existence of weak solutions if $G(x)=1$. Here we refer to their results and adapt them to our problem. Our generalization is not difficult but we prefer to write almost all the details of the proofs in order to make the paper more readable. We consider the (HDD) problem, as derived heuristically in the previous section (we set $2 \varepsilon^{2}=\delta^{2}$ and $\rho=\sqrt{n}$ )

$$
\left\{\begin{array}{l}
\nabla \cdot J=0, \\
J=\rho^{2} \nabla F \\
F=V+h\left(\rho^{2}\right)-G(x) \delta^{2}\left(\frac{\Delta \rho}{\rho}\right)-G^{\prime}(x) \delta^{2}\left(\frac{\nabla \rho}{\rho}\right), \\
-\lambda^{2} \Delta V=\rho^{2}-C,
\end{array}\right.
$$

rewritten in following easier way

$$
\left\{\begin{array}{l}
\nabla \cdot\left(\rho^{2} \nabla F\right)=0 \\
\nabla\left(G(x) \delta^{2} \nabla \rho\right)=\rho\left(V+h\left(\rho^{2}\right)-F\right), \\
-\lambda^{2} \Delta V=\rho^{2}-C
\end{array}\right.
$$

with boundary conditions

$$
\begin{cases}\rho=\rho_{D}, V=V_{D}, F=F_{D} & \text { on } \Gamma_{D} \\ \frac{\partial \rho}{\partial \gamma}=\frac{\partial V}{\partial \gamma}=\frac{\partial F}{\partial \gamma}=0 & \text { on } \Gamma_{N} .\end{cases}
$$

We set the following assumptions:

(A1) $G(x)$ is a strictly positive and continuous function, defined in (1.3);

(A2) $n_{D}, V_{D}, F_{D}, C \in L^{\infty}(\Omega) \cap H^{1}(\Omega), \inf \left(n_{D}\right)>0$. Moreover, there exists $x_{0} \in \Gamma_{D}$ such that $V\left(x_{0}\right)=0$;

(A3) The enthalpy function $h(s)(s \geq 0)$ is strictly monotone increasing, locally Lipschitz continuous, and

$$
\lim _{s \rightarrow 0+} h(s)=-\infty, \quad \lim _{s \rightarrow+\infty} h(s)=+\infty .
$$

We remark that in isothermal condition $h\left(\rho^{2}\right)=2 T \ln \rho$ and the condition (A3) is verified.

\subsection{Approximating problem}

Now we construct a map that solves the Poisson equation. 
Consider $f \in L^{2}(\Omega)$, and set $\Phi \in L^{\infty}(\Omega) \cap H^{1}(\Omega)$ the unique weak solution to the following boundary values problem (BVP)

$$
\left\{\begin{array}{l}
-\lambda^{2} \Delta \Phi=f, \\
\Phi=0 \text { on } \Gamma_{D}, \quad \frac{\partial \Phi}{\partial \gamma}=0 \quad \text { on } \Gamma_{N} .
\end{array}\right.
$$

Let $\Phi_{e} \in L^{\infty}(\Omega) \cap H^{1}(\Omega)$ be the unique weak solution of

$$
\left\{\begin{array}{l}
\Delta \Phi_{e}=0 \\
\Phi_{e}=V_{D} \text { on } \Gamma_{D}, \quad \frac{\partial \Phi_{e}}{\partial \gamma}=0 \quad \text { on } \Gamma_{N}
\end{array}\right.
$$

We define a continuous linear mapping $\tilde{\Phi}[f]=\Phi$ in the following way.

Given $\rho \in L^{2}(\Omega)$, then

$$
V=\tilde{\Phi}\left[\rho^{2}-C\right]+\Phi_{e}
$$

is the unique weak solution of

$$
\left\{\begin{array}{l}
-\Delta \lambda^{2} V=\rho^{2}-C, \\
V=V_{D} \quad \text { on } \Gamma_{D}, \quad \frac{\partial V}{\partial \gamma}=0 \quad \text { on } \Gamma_{N} .
\end{array}\right.
$$

Let $B=\left\{f \in L^{2}(\Omega) \mid \inf F_{D} \leq f \leq \sup F_{D}\right\}$. Given $F \in B, \eta \in(0, \infty)$, for all $\rho \in L^{2}(\Omega)$, define the energy $E_{\eta}(\rho)$,

$$
\begin{aligned}
E_{\eta}(\rho) & =\int_{\Omega} \bar{\delta}^{2}|\nabla \rho|^{2} \mathrm{~d} x+\int_{\Omega} H_{\eta}\left(\rho^{2}\right) \mathrm{d} x+\frac{\lambda^{2}}{2} \int_{\Omega}\left|\tilde{\Phi}_{x}\left[\rho^{2}-C\right]\right|^{2} \mathrm{~d} x \\
& +\int_{\Omega} \rho^{2} \Phi_{e} \mathrm{~d} x-\int_{\Omega} F \rho^{2} \mathrm{~d} x
\end{aligned}
$$

where $H_{\eta}(s)=\int_{1}^{s} h_{\eta}(u) \mathrm{d} u, h_{\eta}(u)=\max \{h(u), h(\eta)\}$ and $\bar{\delta}=G(x) \delta$.

Lemma 3.1. Let $\rho_{c} \in L^{\infty}(\Omega) \cap H^{1}(\Omega)$ and $\rho_{c}=\rho_{D}$ on $\Gamma_{D}$. Define the set $\chi:=\rho_{c}+H_{0}^{1}(\Omega)$. For all given $F \in B$, we set $V=\Phi\left[\rho^{2}-C\right]+\Phi_{e}$. If there exists a minimizer $\rho_{\eta}$ of $E_{\eta}(\rho)$ in $\chi$, then $\rho_{\eta}$ is a weak solution of the following $B V P$

$$
\left\{\begin{array}{l}
-\nabla\left(\bar{\delta}^{2} \nabla \rho\right)=\rho\left(V+h_{\delta}\left(\rho^{2}\right)-F\right), \\
\rho=\rho_{D} \quad \text { on } \Gamma_{D}, \quad \frac{\partial \rho}{\partial \gamma}=0 \quad \text { on } \Gamma_{N} .
\end{array}\right.
$$


Proof. For all $\varphi \in H_{0}^{1}(\Omega), \rho \in \chi$, and $s \in \mathbb{R}$ such that $\rho+s \varphi \in \chi$, we have

$$
\begin{aligned}
E_{\eta}(\rho+s \varphi)-E_{\eta}(\rho) & =\int_{\Omega} \bar{\delta}^{2}|\nabla \rho+s \nabla \varphi|^{2} \mathrm{~d} x-\int_{\Omega} \bar{\delta}^{2}|\nabla \rho|^{2} \mathrm{~d} x \\
& +\int\left(H_{\eta}\left((\rho+s \varphi)^{2}\right)-H_{\eta}\left(\rho^{2}\right)\right) \mathrm{d} x \\
& +\frac{\lambda^{2}}{2} \int_{\Omega}\left(\left|\nabla \tilde{\Phi}\left[(\rho+s \varphi)^{2}-C\right]\right|^{2}-\left|\nabla \tilde{\Phi}\left[\rho^{2}-C\right]\right|^{2}\right) \mathrm{d} x \\
& +\left(\int_{\Omega}(\rho+s \varphi)^{2} \Phi_{e} \mathrm{~d} x-\int_{\Omega} \rho^{2} \Phi_{e} \mathrm{~d} x\right) \\
& -\left(\int_{\Omega} F(\rho+s \varphi)^{2} \mathrm{~d} x-\int_{\Omega} F \rho^{2} \mathrm{~d} x\right) \\
& =I_{1}+I_{2}+I_{3}+I_{4}+I_{5} .
\end{aligned}
$$

Following [7]

$$
\begin{aligned}
& I_{2}=\int_{\Omega}\left(\int_{\rho^{2}}^{(\rho+s \varphi)^{2}} h_{\eta}(u) \mathrm{d} u\right) \mathrm{d} x=\int_{\Omega} 2 \rho s \varphi h_{\eta}\left(\rho^{2}\right) \mathrm{d} x+o(s), \\
& I_{3}=2 \int_{\Omega} \tilde{\Phi}\left[\rho^{2}-C\right] \rho s \varphi \mathrm{d} x+o(s), \\
& I_{4}=\int_{\Omega} 2 \rho s \varphi \Phi_{e} \mathrm{~d} x+o(s) \quad \text { and } \quad I_{5}=\int_{\Omega} 2 \rho s \varphi F \mathrm{~d} x+o(s) .
\end{aligned}
$$

Easily we obtain

$$
\begin{aligned}
I_{1} & =\int_{\Omega} \bar{\delta}^{2}\left(|\nabla \rho|^{2}+s^{2}|\nabla \varphi|^{2}+2 \nabla \rho \cdot s \nabla \varphi\right) \mathrm{d} x-\int_{\Omega} \bar{\delta}^{2}|\nabla \rho|^{2} \mathrm{~d} x \\
& =\int_{\Omega} 2 \bar{\delta}^{2} \nabla \rho \cdot s \nabla \varphi_{x} \mathrm{~d} x+o(s) .
\end{aligned}
$$

So we have

$$
E_{\eta}(\rho+s \varphi)-E_{\eta}(\rho)=2\left(\int_{\Omega} \bar{\delta}^{2} \nabla \rho \cdot s \nabla \varphi \mathrm{d} x+\int_{\Omega} \rho\left(h_{\eta}+V-F\right) s \varphi \mathrm{d} x\right)+o(s) .
$$

If $\rho_{\eta}$ is a minimizer of $E_{\eta}(\rho)$ in $\chi$, then, assuming $s$ tending to 0 , we have:

$$
\begin{aligned}
& E_{\eta}\left(\rho_{\eta}+s \varphi\right)-E_{\eta}\left(\rho_{\eta}\right)= \\
& 2\left(\int_{\Omega} \bar{\delta}^{2} \nabla \rho_{\eta} \cdot s \nabla \varphi \mathrm{d} x+\int_{\Omega} \rho_{\eta}\left(h_{\eta}+V-F\right) s \varphi \mathrm{d} x\right)+o(s) \geq 0 .
\end{aligned}
$$

Let $s \rightarrow 0+$ and $s \rightarrow 0-$, respectively. Then

$$
\int_{\Omega} \bar{\delta}^{2} \nabla \rho_{\eta} \cdot \nabla \varphi \mathrm{d} x+\int_{\Omega} \rho_{\eta}\left(h_{\eta}+V-F\right) \varphi \mathrm{d} x=0 .
$$

Lemma 3.2 (Pseudo-convexity inequality). For all $s \in(0,1), \rho_{1}, \rho_{2} \in L^{2}(\Omega)$ and $\left|\rho_{1}\right| \neq\left|\rho_{2}\right|$, one has $E_{\eta}\left(\sqrt{s \rho_{1}^{2}+(1-s) \rho_{2}^{2}}\right)<s E_{\eta}\left(\rho_{1}\right)+(1-s) E_{\eta}\left(\rho_{2}\right)$. 
We omit the details, we just remark that

$$
|\nabla \rho|^{2} \leq s\left|\nabla \rho_{1}\right|^{2}+(1-s)\left|\nabla \rho_{2}\right|^{2},
$$

which obviously implies

$$
Q(x)|\nabla \rho|^{2} \leq Q(x)\left(s\left|\nabla \rho_{1}\right|^{2}+(1-s)\left|\nabla \rho_{2}\right|^{2}\right) .
$$

Then the thesis follows [7].

Lemma 3.3. Given $F \in B$, there exists a unique non-negative minimizer of $E_{\eta}(\rho)$ in $\chi$.

Proof. We prove the existence of minimizers of $E_{\eta}(\rho)$. First we remark

$$
\lim _{u \rightarrow+\infty} h_{\eta}(u)=+\infty
$$

and then $E_{\eta}$ is coercive with respect to the $L_{2}(\Omega)$ norm. Second we prove the $H^{1}(\Omega)$-weakly sequentially lower semi-continuity of $E_{\eta}(\rho)$. The set $\chi$ is a translate of a Hilbert space, thus the existence of minimizers of $E_{\eta}(\rho)$ follows from the previous analysis. By using Lemma 3.2 we get that, when $\left|\rho_{1}\right| \neq\left|\rho_{2}\right|$, we have

$$
E_{\eta}\left(\sqrt{\frac{1}{2} \rho_{1}^{2}+\frac{1}{2} \rho_{2}^{2}}\right)<\frac{1}{2} E_{\delta}\left(\rho_{1}\right)+\frac{1}{2} E_{\eta}\left(\rho_{2}\right) .
$$

This implies the uniqueness of non-negative minimizer.

We know that, given $F \in L^{\infty}(\Omega), \eta \in(0,+\infty)$, there exists a unique solution $\left(V_{\eta}, \rho_{\eta}\right)$ satisfying $V_{\eta} \in L^{\infty}(\Omega) \cap H^{1}(\Omega)$, and solution of the following problem

$$
\left\{\begin{array}{l}
\nabla\left(\bar{\delta}^{2} \nabla \rho\right)=\rho\left(V+h_{\delta}\left(\rho^{2}\right)-F\right), \\
-\lambda^{2} \Delta V=\rho^{2}-C, \\
\rho=\rho_{D}, V=V_{D} \quad \text { on } \Gamma_{D} ; \quad \frac{\partial \rho}{\partial \gamma}=\frac{\partial V}{\partial \gamma}=0 \quad \text { on } \Gamma_{N} .
\end{array}\right.
$$

In the following result we prove that the charge density $\rho_{\eta}$ is positively defined and bounded by a constant $c$ that does not depend on $\eta$.

Lemma 3.4. Let (A1), (A2) and (A3) hold. Given $F \in L^{\infty}(\Omega)$, then there exists $\eta_{0}>0$ such that, for all $\eta \in\left(0, \eta_{0}\right)$, there exists a unique solution $\left(V_{\eta}, \rho_{\eta}\right)$ to (3.3) which satisfies $V_{\eta} \in L^{\infty}(\Omega) \cap H^{1}(\Omega), \rho_{\eta} \in H^{1}(\Omega), \rho_{\eta}>c$ where $c>0$ is independent of $\eta$.

Proof. Assuming $h\left(s_{0}\right)=0$, we obtain from the monotonicity of the enthalpy function that for all $\eta \in\left(0, s_{0}\right)$, one has

$$
\int_{s_{0}}^{\rho^{2}} h_{\eta}(u) \mathrm{d} u \geq 0
$$

So we get

$$
H_{\eta}\left(\rho^{2}\right)=\int_{1}^{\rho^{2}} h_{\eta}(u) \mathrm{d} u \geq \int_{1}^{s_{0}} h_{\eta}(u) \mathrm{d} u \geq\left(s_{0}-1\right) h_{\eta}(1) \geq\left(s_{0}-1\right) h(1) .
$$


We note that $E_{\eta}(\rho)$ depends on $\eta$ because of $H_{\eta}$. We know that $H_{\eta}$ is bounded from below uniformly for $\eta$ so that $E_{\eta}(\rho)$ is also bounded from below uniformly for $\eta$ in $\chi$.

Now we prove $\rho_{\eta}>c$ where $c>0$ is independent from $\eta$. Using $\left(\rho_{\eta}-c\right)^{-}=$ $\min \left\{0, \rho_{\eta}-c_{q}\right\}$ as a test function for the first equation of (3.3) for $0<c \leq \inf \left(\rho_{D}\right)$, and using the integration by parts, we get

$$
\begin{aligned}
\int_{\Omega} \bar{\delta}^{2}\left|\nabla\left(\left(\rho_{\eta}-c\right)^{-}\right)\right|^{2} \mathrm{~d} x & =\int_{\Omega} \rho_{\eta}\left(V_{\delta}+h_{\delta}\left(\rho_{\eta}^{2}\right)-F\right)\left(-\left(\rho_{\eta}-c\right)^{-}\right) \mathrm{d} x \\
& \leq \int_{\Omega} \rho_{\eta}\left(V_{\eta}+h_{\eta}\left(c^{2}\right)-F\right)\left(-\left(\rho_{\eta}-c\right)^{-}\right) \mathrm{d} x .
\end{aligned}
$$

From $\lim _{s \rightarrow 0+} h(s)=-\infty$, there exists $\left(c_{q}, \bar{\eta}\right)$, with $c>0, \bar{\eta}>0$, such that for $0<\eta \leq \bar{\eta}$, we get

$$
\int_{\Omega} \bar{\delta}^{2}\left|\nabla\left(\left(\rho_{\eta}-c_{q}\right)^{-}\right)\right|^{2} \mathrm{~d} x \leq 0 .
$$

Hence, $\rho_{\eta} \geq c$ when $0<\eta \leq \bar{\eta}$. Set $\eta_{0}=\min \left\{\bar{\eta}, c^{2}\right\}$. Then, when $\eta<\eta_{0}$, it holds $h\left(\rho_{\eta}^{2}\right) \geq h(\eta)$, which implies that trunked enthalpy function does not depend on $\eta$, namely

$$
h_{\eta}\left(\rho_{\eta}^{2}\right)=h\left(\rho_{\eta}^{2}\right)
$$

So $\left\|\rho_{\eta}\right\|_{H^{1}(\Omega)} \leq c_{1}$, with $c_{1}>0$ independent of $\delta$.

From the second equation of (3.3), we obtain $\left\|V_{\eta}\right\|_{L^{\infty}(\Omega)} \leq c_{2}$, and $c_{2}>0$ is independent of $\eta$.

In order to do that, we consider the function $V_{c} \in L^{\infty}(\Omega) \cap H^{1}(\Omega)$ and $V_{c}=V_{D}$ on $\Gamma_{D}$, and we use $V-V_{c}$ as test function in the second equation in (3.3)

$$
\begin{aligned}
& \lambda^{2} \int_{\Omega}\left|\nabla V_{\eta}\right|^{2} \mathrm{~d} x+\lambda^{2} \int_{\Omega} \nabla V_{c} \cdot \nabla V_{\eta} \mathrm{d} x=\int_{\Omega}\left(\rho_{\eta}^{2}-C\right)\left(V_{\eta}-V_{c}\right) \mathrm{d} x \\
\leq & \int_{\Omega}\left|\left(\rho_{\eta}^{2}-C\right) V_{c}\right| \mathrm{d} x+\frac{1}{\lambda^{2}} \int_{\Omega}\left|\rho_{\eta}^{2}-C\right|^{2} \mathrm{~d} x+\frac{\lambda^{2}}{4} \int_{\Omega}\left|V_{\eta}\right|^{2} \mathrm{~d} x .
\end{aligned}
$$

The result follows assuming the Poincaré inequality, and the Sobolev's Immersion Theorem taking into account $\left\|\rho_{\eta}\right\|_{H^{1}(\Omega)} \leq c_{1}$ and the assumption (A2).

Lemma 3.4 immediately implies the following result.

Lemma 3.5. Let (A1), (A2) and (A3) hold, given $F \in L^{\infty}(\Omega)$, there exists a unique solution $(V, \rho)$, with $V \in L^{\infty}(\Omega) \cap H^{1}(\Omega), \rho \in H^{1}(\Omega)$, to the BVP

$$
\left\{\begin{array}{l}
\nabla\left(\bar{\delta}^{2} \nabla \rho\right)=\rho\left(V+h\left(\rho^{2}\right)-F\right), \\
-\lambda^{2} \Delta V=\rho^{2}-C, \\
\rho=\rho_{D}, V=V_{D} \quad \text { on } \Gamma_{D} ; \quad \frac{\partial \rho}{\partial \gamma}=\frac{\partial V}{\partial \gamma}=0 \quad \text { on } \Gamma_{N} .
\end{array}\right.
$$

Lemma 3.6. Let $\rho$ be the solution in Lemma 3.5. Then $\rho \in L^{\infty}(\Omega)$.

Proof. Now we prove that $\rho$ is bounded from above. Using

$$
(\rho-a)^{+}=\max \{0, \rho-a\}
$$


as a test function in the first equation of (3.4), for some $a \geq \sup _{\Gamma_{D}} \rho_{D}$ with $\rho_{D}>0$ to be determined, we get

$$
\int_{\Omega} \bar{\delta}^{2}\left|\nabla\left((\rho-a)^{+}\right)\right|^{2} \mathrm{~d} x=\int_{\Omega} \rho\left(F-V-h\left(\rho^{2}\right)\right)(\rho-a)^{+} \mathrm{d} x .
$$

Following from $\lim _{s \rightarrow+\infty} h(s)=+\infty$, we know that there exists a positive constant $a \geq \sup _{\Gamma_{D}} \rho_{D}$ such that when $\rho>a$, one has $F-V-h\left(\rho^{2}\right)<0$. Hence we get

$$
\int_{\Omega} \bar{\delta}^{2}\left|\nabla\left((\rho-a)^{+}\right)\right|^{2} \mathrm{~d} x \leq 0
$$

and then $\rho \leq a$.

\subsection{Existence of weak solutions for the approximating problem}

Theorem 3.7. Let (A1), (A2) and (A3) hold. Then there exists a solution $(\rho, V, F) \in L^{\infty}(\Omega) \cap H^{1}(\Omega)$ to problem (3.1) with the boundary conditions (3.2).

Proof. We set $F=f \in B$ in the second equation of (3.1) to get

$$
\left\{\begin{array}{l}
\nabla \cdot\left(\rho^{2} \nabla F\right)=0, \\
\nabla\left(\bar{\delta}^{2} \nabla \rho\right)=\rho\left(V+h\left(\rho^{2}\right)-f\right), \\
-\lambda^{2} \Delta V=\rho^{2}-C .
\end{array}\right.
$$

There exists a unique solution $(\rho, V, F) \in\left(L^{\infty}(\Omega) \cap H^{1}(\Omega)\right)^{3}$ of (3.5) satisfying the boundary conditions

$$
\begin{cases}\rho=\rho_{D}, V=V_{D}, F=F_{D} & \text { on } \Gamma_{D} \\ \frac{\partial \rho}{\partial \gamma}=\frac{\partial V}{\partial \gamma}=\frac{\partial F}{\partial \gamma}=0 & \text { on } \Gamma_{N} .\end{cases}
$$

Using the maximum principle we get

$$
\inf F_{D} \leq F \leq \sup F_{D}
$$

hence $F \in B$.

Thus the mapping $T: B \rightarrow B, T(f)=F$ is well defined. We find $\rho$ and $V$ from the second and third equation of (3.5), with fixed $f \in B$, and then, by using the first equation, we obtain a new function $f \in B$. It is not difficult to check that $T$ is compact, because of the compact embedding $H^{1}(\Omega) \hookrightarrow L^{2}(\Omega)$. Now we need to prove that $T$ is continuous. Following [7], we assume $f_{n} \in B$ is convergent to $f$ as $n \rightarrow \infty$ in $L^{2}(\Omega),(\rho, F, V) \in\left(L^{\infty}(\Omega) \cap H^{1}(\Omega)\right)^{3}$ solution to (3.5), (3.2), and

$$
\left(\rho^{(n)}, F^{(n)}, V^{(n)}\right) \in\left(L^{\infty}(\Omega) \cap H^{1}(\Omega)\right)^{3}
$$

solution to (3.5)-(3.2) with $f_{n}$ instead of $f$. Then we replace $F$ with $f_{n}$ in the definition of $E_{\delta}(\rho)$ and define it as $E_{\delta}^{(n)}(\rho)$. Then we have

$$
\lim _{n \rightarrow+\infty} E_{\delta}^{(n)}(\rho)=E_{\delta}(\rho) .
$$

Thus one has

$$
\lim _{n \rightarrow+\infty} \inf _{\rho \in \chi} E_{\delta}^{(n)}(\rho)=\inf _{\rho \in \chi} E_{\delta}(\rho) .
$$


$E_{\delta}(\rho)$ is continuous, so we get from Lemma 3.3

$$
\lim _{n \rightarrow+\infty}\left\|\rho^{(n)}-\rho\right\|=0 .
$$

Now we use the first equation in (3.5) and we get

$$
\lim _{n \rightarrow+\infty}\left\|F^{(n)}-F\right\|=0 .
$$

Hence $T$ is continuous. The results follow by using Schauder fixed-point theorem.

\subsection{Hybrid limit}

The aim of this section is to prove the existence of weak solutions, when the quantum effect is negligible in a subset of our domain. In other words we will extend the results discussed in the previous section even to the hybrid case. Assume $G_{\alpha}(x)$ as defined in (1.3), and $h(n)=\ln n$. We consider the system

$$
\left\{\begin{array}{l}
\nabla \cdot\left(\rho_{\alpha} \nabla F_{\alpha}\right)=0, \\
\delta^{2} \nabla\left(G_{\alpha}(x) \nabla \rho_{\alpha}\right)=\rho\left(V_{\alpha}+2 \ln \rho_{\alpha}-F_{\alpha}\right), \\
-\lambda^{2} \Delta V_{\alpha}=\rho_{\alpha}^{2}-C
\end{array}\right.
$$

with boundary conditions

$$
\begin{cases}\rho_{\alpha}=\sqrt{n_{D}^{\alpha}}, \quad V_{\alpha}=V_{D}^{\alpha}, \quad F_{\alpha}=F_{D}^{\alpha} & \text { on } \Gamma_{D} \\ \frac{\partial \rho_{\alpha}}{\partial \gamma}=\frac{\partial V_{\alpha}}{\partial \gamma}=\frac{\partial F_{\alpha}}{\partial \gamma}=0 & \text { on } \Gamma_{N} .\end{cases}
$$

Of course $n, V$ and $F$ as well depend on the parameter $\alpha$, as $\alpha \rightarrow 0+$, so we have $\left(\rho_{m}, V_{m}, F_{m}\right) \rightarrow(\rho, V, F)$, where $(\rho, V, F)$ is the solution to the limit problem, that is

$$
\left\{\begin{array}{l}
\nabla \cdot(\rho \nabla F)=0, \\
\delta^{2} \nabla(H(x) \nabla \rho)=\rho(V+2 \ln \rho-F) \\
-\lambda^{2} \Delta V=\rho^{2}-C
\end{array}\right.
$$

with boundary conditions

$$
\begin{cases}\rho=\sqrt{n_{D}}, \quad V=V_{D}, \quad F=F_{D} & \text { on } \Gamma_{D} \\ \frac{\partial \rho}{\partial \gamma}=\frac{\partial V}{\partial \gamma}=\frac{\partial F}{\partial \gamma}=0 & \text { on } \Gamma_{N},\end{cases}
$$

We set the following assumptions:

(A4) $n_{D}^{\alpha}, V_{D}^{\alpha}, F_{D}^{\alpha}, \inf n_{D}>0$;

(A5) $F_{D}^{\alpha}$ is uniformly bounded in $\alpha$;

(A6) $n_{D}^{\alpha} \rightarrow n_{D}, V_{D}^{\alpha} \rightarrow V_{D}, F_{D}^{\alpha} \rightarrow F_{D}$ in $H^{1}(\Omega)$ as $\alpha \rightarrow 0+$.

Theorem 3.8. Assume (A1)-(A6). There exists

$$
(\rho, F, V) \in L^{\infty}(\Omega) \cap H^{1}(\Omega)
$$


solution to (3.6)-(3.7), and $\rho_{\alpha} \rightarrow \rho, F_{\alpha} \rightarrow F, V_{\alpha} \rightarrow V$ weakly in $H^{1}(\Omega)$ and strongly in $L^{2}$ as $\alpha \rightarrow 0+$.

Proof. Following [7], we know there exist $\rho, V, F \in L^{\infty}(\Omega) \cap H^{1}(\Omega)$ satisfying (3.6)-(3.7) where $\rho \in \chi$ is the unique minimizer of $\tilde{E}(\rho)$ :

$$
\widetilde{E}(\rho)=\delta^{2} \int_{\Omega_{q}}|\nabla \rho|^{2} \mathrm{~d} x+\int_{\Omega}\left|\nabla \tilde{\Phi}\left[\rho^{2}-C\right]\right| \mathrm{d} x+\int_{\Omega} \rho^{2}\left(\Phi_{e}-F\right) \mathrm{d} x+\int_{\Omega} H\left(\rho^{2}\right) \mathrm{d} x .
$$

Now we consider the functional

$E_{\alpha}(\rho)=\delta^{2} \int_{\Omega} G_{\alpha}(x)|\nabla \rho|^{2} \mathrm{~d} x+\int_{\Omega}\left|\nabla \tilde{\Phi}\left[\rho^{2}-C\right]\right| \mathrm{d} x+\int_{\Omega} \rho^{2}\left(\Phi_{e}-F_{\alpha}\right) \mathrm{d} x+\int_{\Omega} H\left(\rho^{2}\right) \mathrm{d} x$,

and $\rho_{\alpha}$ is the unique minimizer of (3.8) in $\Upsilon=\rho_{D}+H_{0}^{1}(\Omega)$. Because of the maximum principle, $F_{\alpha}$ is bounded and its bound does not depend on $\alpha$. It is not difficult to prove that also $\rho_{\alpha}$ and $V_{\alpha}$ are uniformly bounded in $\alpha$. Then there exists a subsequence of $\rho_{\alpha}, F_{\alpha}, V_{\alpha}$ such that

$$
\rho_{\alpha} \rightarrow \rho^{*}, \quad F_{\alpha} \rightarrow F^{*}, \quad V_{\alpha} \rightarrow V^{*}
$$

weakly in $H^{1}(\Omega)$ and strongly in $L^{2}(\Omega)$ as $\alpha \rightarrow 0+$.

We know $\rho^{*} \in \Upsilon$ and

$$
\limsup _{\alpha \rightarrow 0+} E_{\alpha}\left(\rho_{\alpha}\right) \leq \limsup _{\alpha \rightarrow 0+} E_{\alpha}\left(\rho^{*}\right) \leq \widetilde{E}\left(\rho^{*}\right)
$$

and

$$
E_{\alpha}\left(\rho_{\alpha}\right)=\tilde{E}\left(\rho_{\alpha}\right)+\int_{\Omega} \delta^{2}\left(G_{\alpha}-H\right)(x)|\nabla \rho|^{2} \mathrm{~d} x+\int_{\Omega}\left(F-F_{\alpha}\right) \mathrm{d} x .
$$

Then because of the weakly lower semi-continuity of $\widetilde{E}(\rho)$, we conclude

$$
\liminf _{\alpha \rightarrow 0+} E_{\alpha}\left(\rho_{\alpha}\right) \geq \widetilde{E}(\rho),
$$

and, using (3.10), we conclude $\left(F-F_{\alpha}\right) \rightarrow 0$. Taking into account (1.3), finally we get

$$
E_{\alpha}\left(\rho_{\alpha}\right) \rightarrow \widetilde{E}\left(\rho^{*}\right)
$$

if $\alpha \rightarrow 0+$ and then $\rho^{*}$ is the unique minimizer of $\widetilde{E}\left(\rho^{*}\right)$.

Now we need to prove that $\rho^{*}=\rho$. The relations (3.9) and (3.11) imply $\widetilde{E}\left(\rho^{*}\right) \geq \widetilde{E}(\rho)$. However $\rho^{*}$ is the unique minimizer of $\widetilde{E}\left(\rho^{*}\right)$, hence $\widetilde{E}\left(\rho^{*}\right)=\widetilde{E}(\rho)$ and $\rho=\rho^{*}$. Therefore it is easy to prove $F=F^{*}$ and $V=V^{*}$.

\section{Numerical simulations for Hybrid Drift Diffusion}

In this section we present some numerical simulations on electronic devices, by using the Scilab tool for BVP, COLNEW, which is an update of the COLSYS code (see, e.g. [3] or [6] and its bibliography). 
Here we work in the one-dimensional case and from now on $\Omega=[0,1]$. We consider the following system

$$
\left\{\begin{array}{l}
F_{x x}=-u_{x} F_{x} \\
u_{x x}=-\frac{u_{x}^{2}}{2}+\frac{(V+u-F)}{G \delta^{2}}-\frac{u_{x} G^{\prime}}{G}, \\
V_{x x}=\frac{C-\rho^{2}}{\lambda^{2}} .
\end{array}\right.
$$

obtained from (3.1) by setting $\rho=e^{u / 2}$. We solve (4.1) coupled with the following boundary conditions

$$
\left\{\begin{array}{l}
u(0)=\ln C(0), \quad u(1)=\ln C(1), \quad V(0)=0, \quad V(1)=2, \\
(V+u-F)(0)=(V+u-F)(1)=0 .
\end{array}\right.
$$

In our simulations we consider $\lambda=0.01$ and different value of the reduced Plank's constant $\delta$. The first device we consider is a simple $n^{+} n$ diode used in [11], and modeled by the following doping profile

$$
C(x)=0.75-0.25 \tanh (100(x-0.5)) .
$$

In this case we use, as quantum correction function

$$
G(x)=0.75-0.25 \tanh (100(x-0.45)),
$$

which is shifted to the left of 0.05 , with respect the doping profile $C(x)$ (see Figure 1). In Figure 2 and Figure 3, we compare H-DD-QDD and QDD assuming
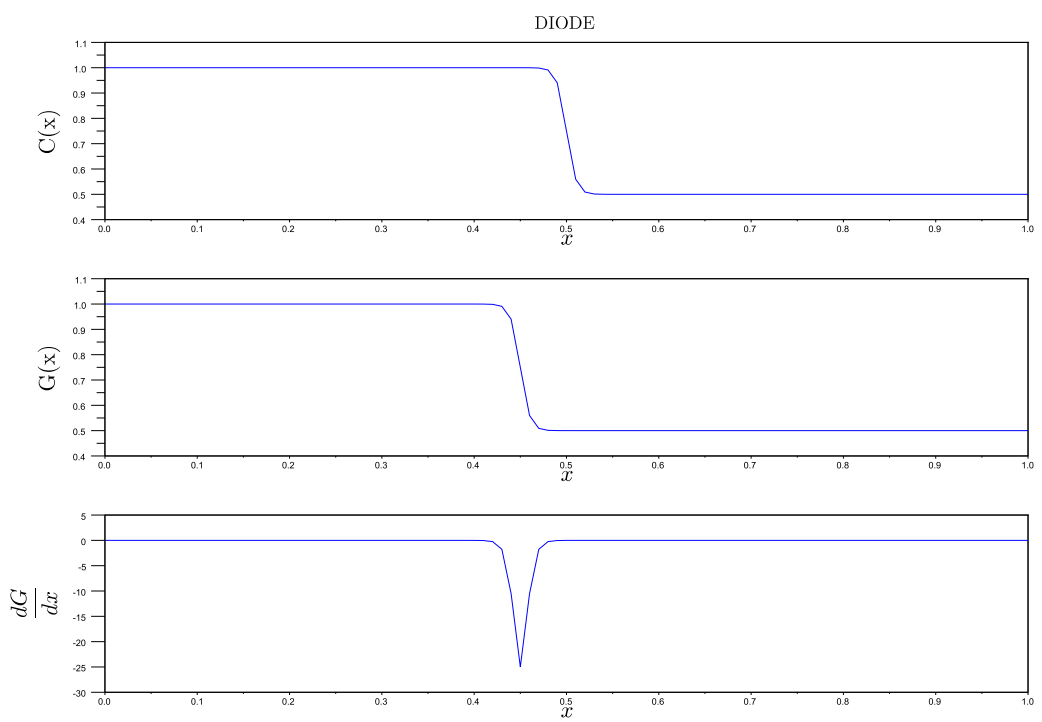

Figure 1. Diode (4.2): Doping profile, quantum correction terms and its derivative with respect to $x$.

$\delta=0.01$. In particular we are interested in the behaviors of charge density, electrostatic potential, generalized chemical potential and current density. Figure 2 
and Figure 3 show there are not remarkable difference in the behavior of these quantities in the hybrid case with respect to the quantum one. The same holds assuming $\delta=0.001$ (see Figure 4 and Figure 5).

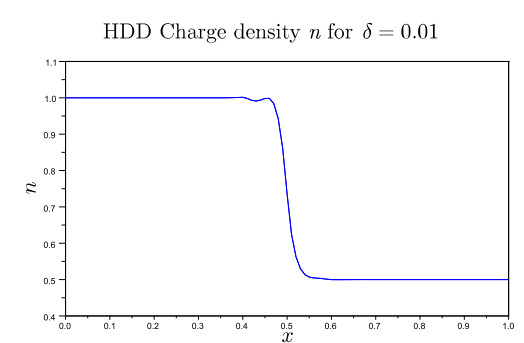

HDD Generalized chemical potential $F$ for $\delta=0.01$

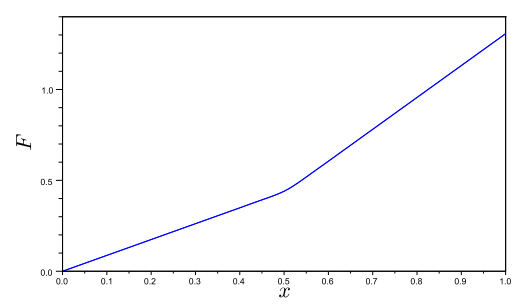

HDD Electrostatic potential $V$ for $\delta=0.01$

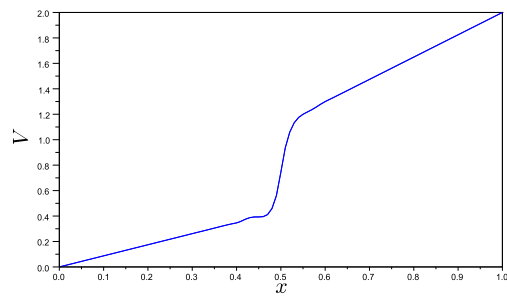

HDD Current density $J$ for $\delta=0.01$

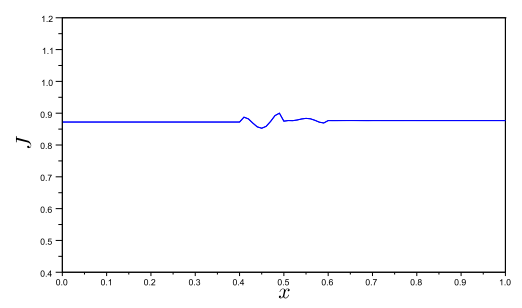

Figure 2. HDD diode with $\delta=0.01$.

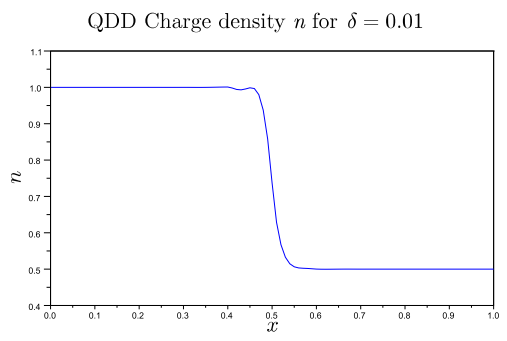

QDD Generalized chemical potential $F$ for $\delta=0.01$

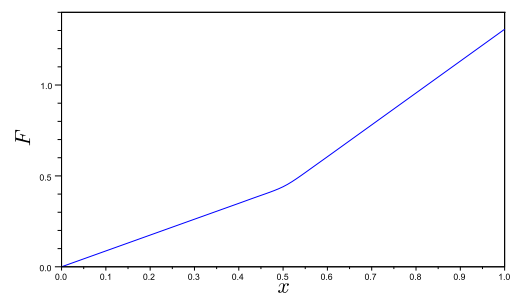

QDD Electrostatic potential $V$ for $\delta=0.01$

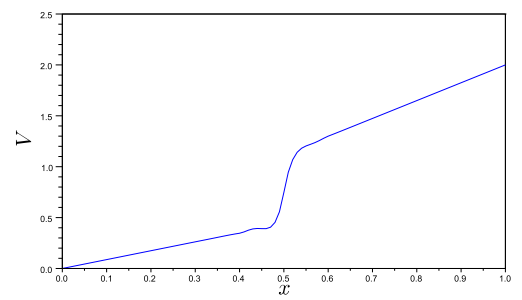

QDD Current density $J$ for $\delta=0.01$

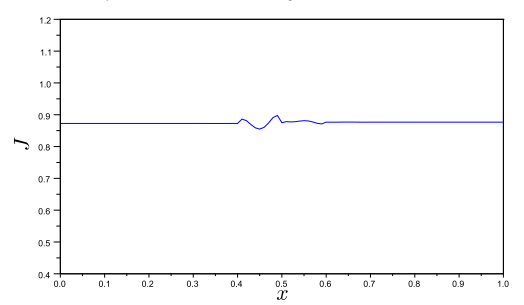

Figure 3. QDD diode with $\delta=0.01$.

The second device we take into account is a resonant tunnel diode (RTD), studied by Degond et al. in [8], by using a coupled Schrödinger-DD stationary model. This device is composed by $5 \mathrm{~nm}$ undoped GaAs quantum well sandwiched 
HDD Charge density $n$ for $\delta=0.001$

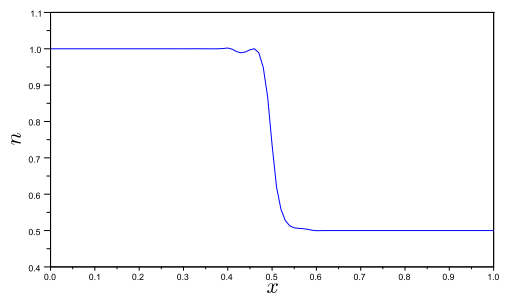

HDD Generalized chemical potential $F$ for $\delta=0.001$

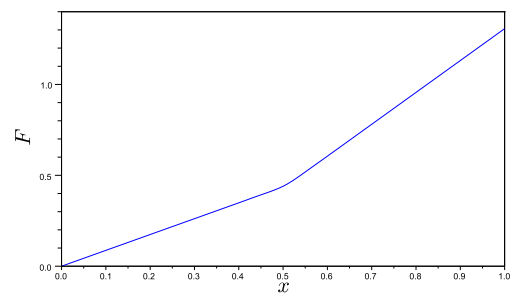

HDD Electrostatic potential $V$ for $\delta=0.001$

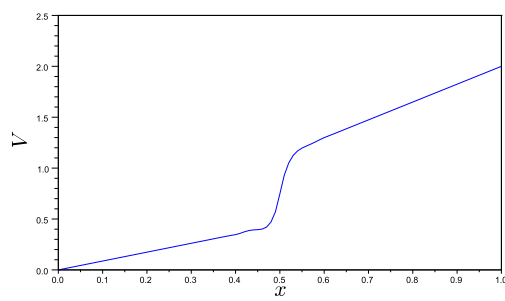

HDD Current density $J$ for $\delta=0.001$

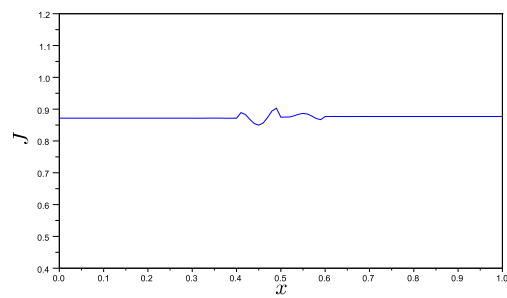

Figure 4. HDD diode with $\delta=0.001$.

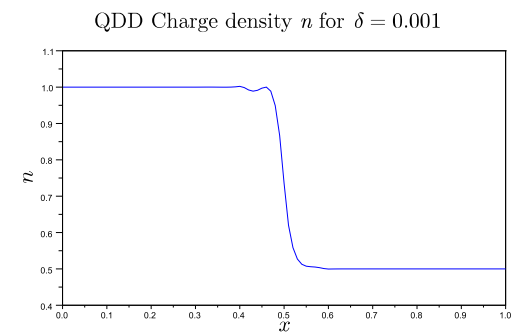

QDD Generalized chemical potential $F$ for $\delta=0.001$

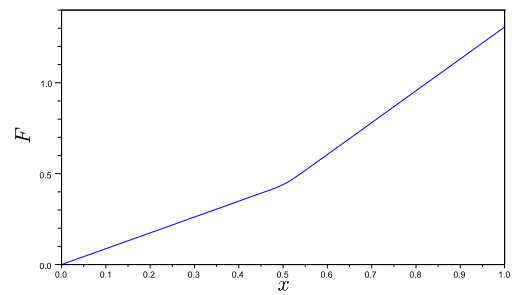

QDD Electrostatic potential $V$ for $\delta=0.001$

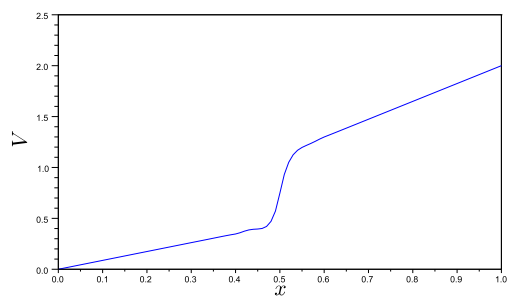

QDD Current density $J$ for $\delta=0.001$

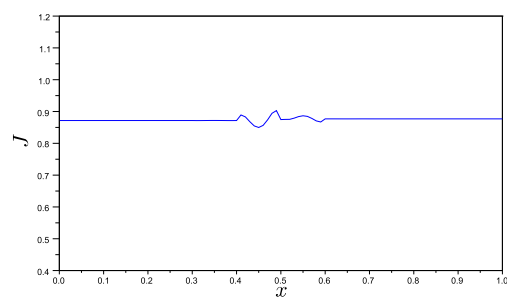

Figure 5. QDD diode with $\delta=0.001$.

between two $5 \mathrm{~nm}$ undoped $A l_{0.3} G a_{0.7} A s$ tunnel barriers. A double-barrier heterostructure is placed between two undoped thin cladding layers $2.5 \mathrm{~nm}$ in width and two $50 \mathrm{~nm}$ GaAs spacer layers with doping densities of $2 \cdot 10^{22} \mathrm{~m}^{-3}$. Each GaAs contact layer is $500 \mathrm{~nm}$ wide with doping densities of $2 \cdot 10^{24} \mathrm{~m}^{-3}$. Its total length is $L=1120 \mathrm{~nm}$. Then we scale these quantities assuming, as reference values for the charge density and the lengths, $C^{*}=2 \cdot 10^{24} \mathrm{~m}^{-3}$ and $L$, respectively. This 
implies, the scaled variables are

$$
n_{s}(0)=n_{s}(1)=1, \quad L_{s}=1 \text {. }
$$

The doping profile and the hybrid correction function are respectively

$$
\begin{aligned}
C(x) & =-0.495 \tanh (1000(x-23 / 56))-0.005 \tanh 10000(x-55 / 112) \\
& +0.005 \tanh 1000(x-57 / 112)+0.495 \tanh 1000(x-33 / 56)+1
\end{aligned}
$$

and

$$
G(x)=0.495 \tanh 1000(x-21 / 56)-0.495 \tanh 1000(x-35 / 56)-0.01 .
$$

In Figure 6 and Figure 7, we compare H-DD-QDD and QDD assuming $\delta=0.01$. These results show that there are not remarkable differences in the behavior of these quantities in the hybrid case with respect the quantum one. The same holds assuming $\delta=0.001$ (see Figure 8 and Figure 9). In addition, our model suggests a less expensive way to simulate complex quantum devices: it provides a coherent description of quantum phenomena wherever necessary, even it behaves in classical way in a large part of the device, where the quantum effects are negligible. In the future we will study a time dependent hybrid model, which is a very interesting and still open problem.

HDD Charge density $\mathrm{n}$ for $\delta=0.01$

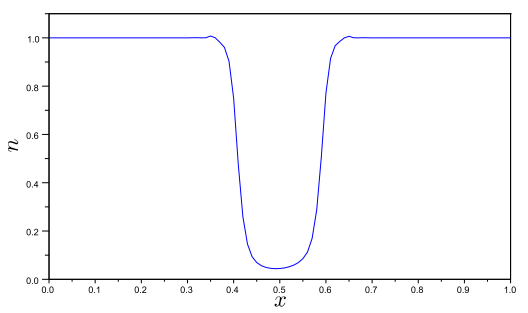

HDD Generalized chemical potential $\mathrm{F}$ for $\delta=0.01$

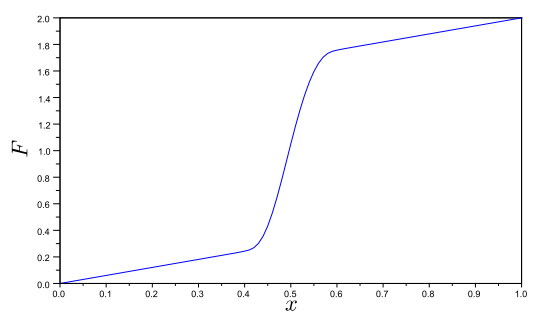

HDD Electrostatic potential $\mathrm{V}$ for $\delta=0.01$

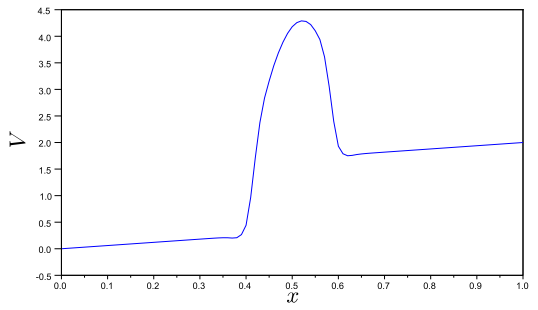

HDD Current density $\mathrm{J}$ for $\delta=0.01$

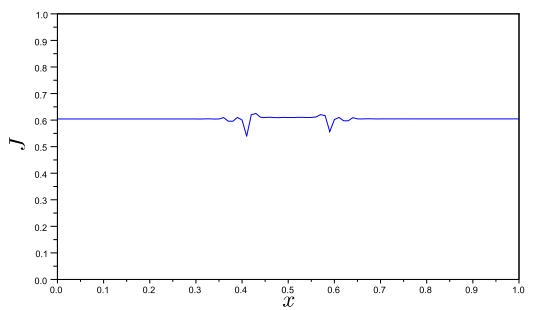

Figure 6. HDD RTD with $\delta=0.01$. 


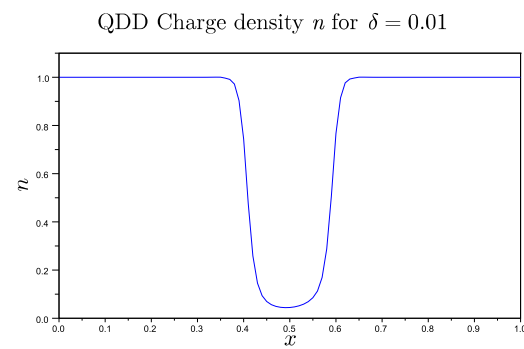

QDD Generalized chemical potential $F$ for $\delta=0.01$

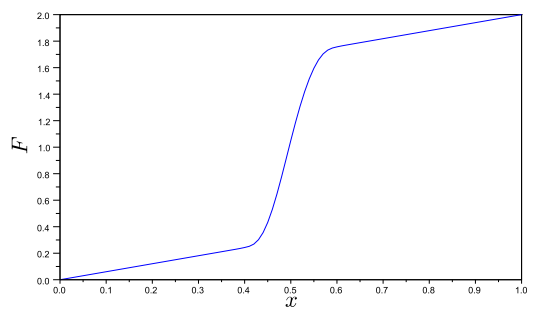

QDD Electrostatic potential $V$ for $\delta=0.01$

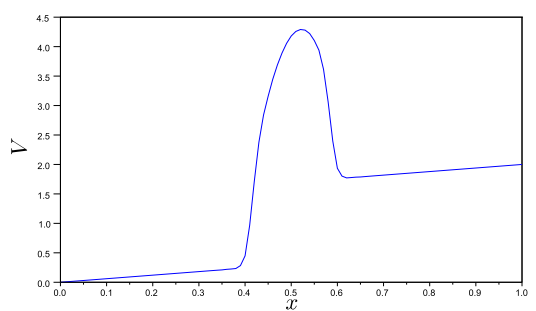

QDD Current density $J$ for $\delta=0.01$

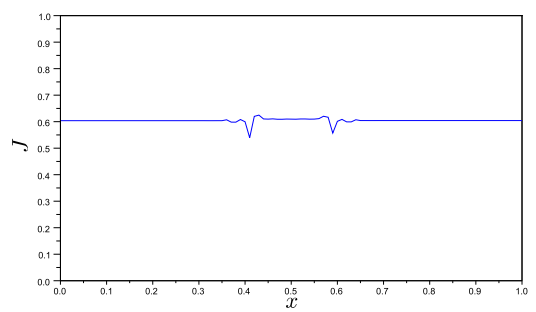

Figure 7. QDD RTD with $\delta=0.01$.

HDD Charge density $n$ for $\delta=0.001$

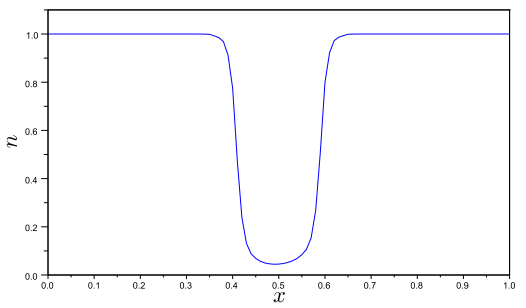

HDD Generalized chemical potential $F$ for $\delta=0.001$

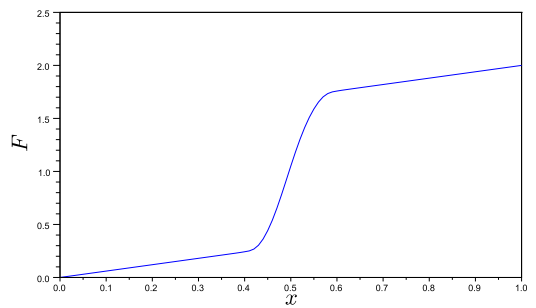

HDD Electrostatic potential $V$ for $\delta=0.001$

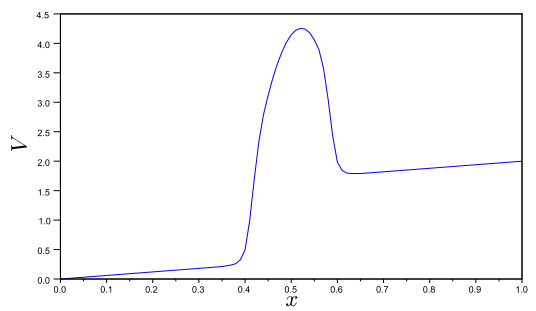

HDD Current density $J$ for $\delta=0.001$

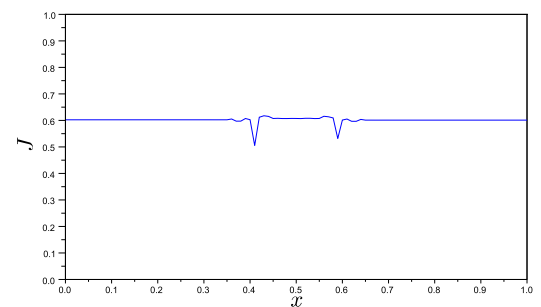

Figure 8. HDD RTD with $\delta=0.001$. 
QDD Charge density $n$ for $\delta=0.001$

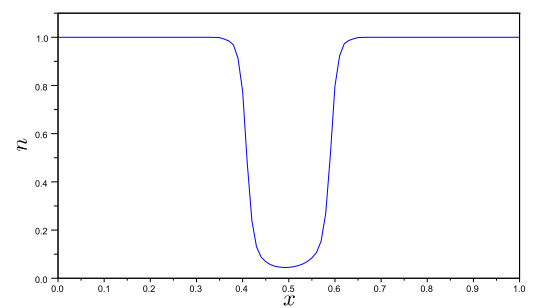

QDD Generalized chemical potential $F$ for $\delta=0.001$

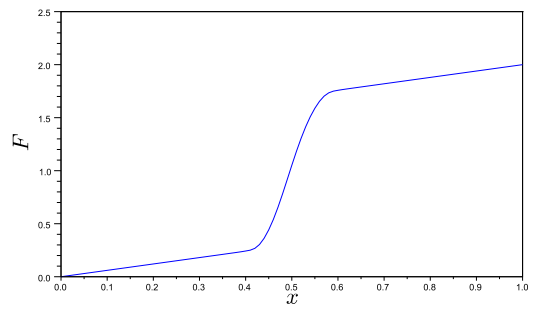

QDD Electrostatic potential $V$ for $\delta=0.001$

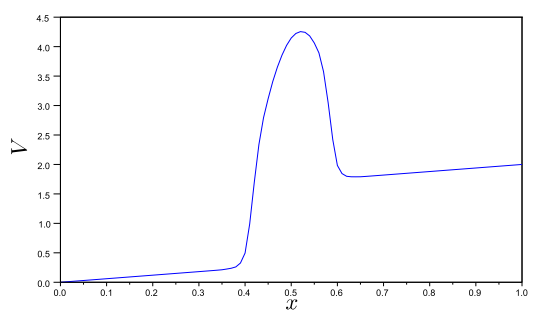

QDD Current density $J$ for $\delta=0.001$

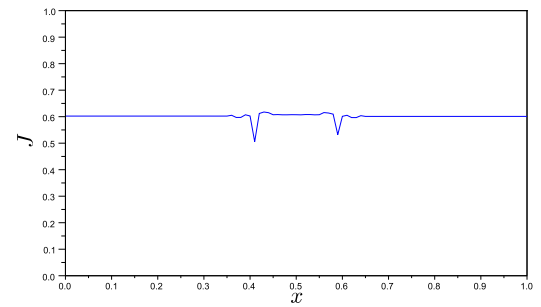

Figure 9. QDD RTD with $\delta=0.001$.

Acknowledgment. The authors thank the referee for recommending various improvements in exposition.

\section{REFERENCES}

[1] M. G. Ancona and G. J. Iafrate, Quantum correction to the equation of state of an electron gas in a semiconductor, Phys. Rev. B 39 (1989), 9536-9540.

[2] M. G. Ancona and H. F. Tiersten, Macroscopic physics of the silicon inversion layer, Phys. Rev. B 35 (1987), 7959-7965.

[3] U. Ascher, J. Christiansen and R. D. Russell, A collocation solver for mixed order systems of boundary value problems, Math. Comp. 33 (1979), 659-679.

[4] M. Baro, N. Ben Abdallah, P. Degond and A. El Ayyadi, A 1 D coupled Schrödinger driftdiffusion model including collisions, J. Comput. Phys. 203 (2005), 129-153.

[5] N. Ben Abdallah, A hybrid kinetic-quantum model for stationary electron transport, J. Stat. Phys. 125 (1998), 309-335.

[6] S. L. Campbell, J.P. Chancelier and R. Nikoukhah, Modeling and Simulation in Scilab/Scicos, Springer, 2009.

[7] Q. Chen and P. Guan, Weak solutions to the stationary quantum drift-diffusion model, J. Math. Anal. Appl. 359 (2009), 666-673.

[8] P. Degond and A. El Ayyadi, A coupled Schrödinger drift-diffusion model for quantum semiconductor device simulations, J. Comput. Phys. 181 (2002), 222-259.

[9] G. L. Gardner, The quantum hydrodynamic model for semiconductor devices, SIAM J. Appl. Math. 54 (1994), 409-427.

[10] A. Jüngel, Transport Equations for Semiconductors, Springer, 2009.

[11] A. Jüngel and H. Li, Quantum Euler-Poisson systems: existence of steady states, Archivum Math. 40 (2004), 435-456.

[12] P. A. Markowich, C.A. Ringhofer and C. Schmeiser, Semiconductors Equations, SpringerVerlag, 1989.

[13] R. Pinnau, The linearized transient quantum drift diffusion model stability of stationary states, Z. Angew. Math. Mech. 80 (2000), 327-344. 
[14] R. Pinnau and A. Unterreiter, The stationary current-voltage characteristics of the quantum drift diffusion model, SIAM J. Numer. Anal. 37 (1999), 211-245.

Simone Chiarelli, Department of Pure and Applied Mathematics, University of L'Aquila, via Vetoio, loc. Coppito - 67010 L'Aquila, Italy

e-mail: simone.chiarelli@mathmods.eu

Federica Di Michele, Department of Mathematics, University of Hamburg, Bundesstrasse 55, 20146 Hamburg, Germany

e-mail: federica.di.michele@math.uni-hamburg.de

Bruno Rubino, Department of Pure and Applied Mathematics, University of L'Aquila, via Vetoio, loc. Coppito - 67010 L'Aquila, Italy

e-mail: bruno.rubino@univaq.it 
